\title{
A mortalidade por câncer de estômago no Brasil: análise do período de 1977 a 1989
}

\author{
Sto mach cancer mortality in Brazil: \\ from 1977 to 1989
}

Maria do Rosario Dias de Oliveira Latorre 1

\footnotetext{
1 Departamento de Epidemiologia, Faculdade de Saúde Pública, Universidade de São Paulo. Av. Dr. Arnaldo 715, São Paulo, SP 01246-904, Brasil. E-mail: mdrddola@usp.br
}

\begin{abstract}
Both incidence and mortality of stomach cancer have been declining in most of the world in the last decades. However, this phenomenon has not been studied in Brazil. Although data on incidence rates are insufficient, there is a standardized historical series on mortality data beginning in 1977. This study analyzes trends for stomach cancer mortality during the 19771989 period for Brazil and Brazilian State capitals, using the standardized mortality rates of stomach cancer (standardized population: Brazil-1980). The results showed that mortality rates have been decreasing in Brazil as a whole and nearly all of the State capitals. However, differences in mortality-rate trends appeared when patterns and location of death were analyzed. The decline was most evident in the Southeast, South, and Center-West, similar to that of European countries. Although stomach cancer is still an important cause of fatal cancer (particularly in males), stomach cancer mortality is currently less important than lung and breast cancer mortality in these regions. Unfortunately, we lack historical inci dence series to compare with mortality data to determine whether they reflect actual trends in the inci dence of stomach cancer in Brazil. Key words Stomach Cancer; Mortality; Trends
\end{abstract}

Resumo Tanto a inci dência quanto a mortalidade por câncer de estômago vêm decl inando, nas últimas décadas, em grande parte do mundo, fenômeno este ai nda não estudado no Brasil. Para a inci dência não existem séries históri cas de tamanho sufi ci ente para se anali sarem tendências com certo grau de confiabilidade. Porém, é possível estudar-se o comportamento dessa doença por meio dos dados de mortalidade. Este trabal ho analisou a tendência da mortalidade por câncer de estômago no Brasil e capitais, no período de 1977 a 1989, através dos coefi cientes de mortalidade por câncer de estômago padronizados, tendo como população padrão a do Brasil (1980). Em que pese a grande di spari dade existente entre as capitais brasil ei ras, a tendência da mortalidade por este tipo de câncer apresentada nesse período é de declínio, variando um pouco a forma com que se deu esse decrésci mo nos coefi ci entes. Nas capitais das Regi ões Sudeste, Sul e Centro-Oeste, o declíni o é mais marcante, e óbi tos por outras local izações de câncer, como pulmão e mama, são, atualmente, mais freqüentes. No entanto, a mortalidade por câncer de estômago ai nda é i mportante como locali zação de câncer, princi palmente entre os homens. As tendências verificadas para esse período mostram-se semel hantes às observadas princi pal mente na Europa elamenta-se não existirem séries históricas da incidência de câncer de estômago, para se avaliar se o decréscimo observado na mortalidade refletea tendência da verdadei ra incidência. Palavras-chave Câncer de Estômago; Mortalidade; Tendência 
Introdução

O padrão da mortalidade no Brasil passou por profundas modificações na última década, porém estas alterações não foram uniformes dentro do País e nem sempre têm acompanhado o que aconteceu em décadas anteriores em países desenvolvidos, onde as doenças cardiovasculares e as neoplasias são as causas de óbito mais importantes.

A Tabela 1 apresenta o número, a mortalidade proporcional por neoplasias e a posição ocupada por esse grupo de doenças na mortalidade geral no Brasil e em suas capitais, em dois anos: 1978 e 1989.

Observa-se que não existe um padrão uniforme da mortalidade. Nas regiões menos desenvolvidas, como algumas capitais das Regiões Norte e Nordeste, a mortalidade infantil ainda é alta e as doenças infecto-contagiosas contribuem com grande porcentagem de óbitos, fazendo com que os cânceres ocupem a quarta ou quinta colocação na mortalidade geral.

Em outras capitais e no Brasil como um todo, são as causas externas que ocupam a segunda posição, deixando as neoplasias em terceiro lugar. Isso deveu-se, fundamentalmente, ao acréscimo nos óbitos por acidentes de trânsito na Região Centro-Oeste e parte da Região Sul (Mello Jorge \& Latorre, 1994) e por homicídios em parte do, Nordeste e Sudeste (Souza, 1994).

Porém é inegável o papel dos cânceres como causa básica de óbito no Brasil. Na maioria das capitais os neoplasmas ocupam o segundo ou terceiro posto, respondendo por $10 \%$ dos óbitos, em média. Em São Paulo, Rio de Janeiro e nos estados do Sul, esta porcentagem está sempre acima da média nacional. Comparando-se os números absolutos, no Brasil houve um aumento de, aproximadamente, $47 \%$ nos óbitos por câncer.

O fenômeno de aumento da mortalidade por câncer é observado na maioria dos países, geralmente devido, principalmente, ao acréscimo da mortalidade pelo câncer do pulmão (categoria 162 da CID 9ạ revisão). Ao mesmo tempo, tem ocorrido um fato surpreendente e ainda não totalmente explicado: o declínio da mortalidade por câncer de estômago (categoria 151 da CID 9ạ revisão). Esse decréscimo acontece, também, na incidência desta localização em grande parte do mundo (Coleman et al., 1993), embora ainda seja um câncer muito letal, não tendo sido observadas mudanças nas taxas de sobrevida. Howson et al. (1986) e Haenzel \& Correa (1975) referem que as expli- cações para esse declínio, muitas vezes marcantes, ainda são hipóteses, porém devem estar relacionadas à introdução da refrigeração, mudanças nas técnicas de preservação de alimentos (utilização de menos sal e alimentos menos condimentados) e ao maior consumo de frutas e vegetais frescos. Além disso, acredita-se que a queda deva estar ocorrendo, principalmente, no tipo intestinal (epidêmico) - que é mais prevalente - e não no difuso. Porém, não existem séries históricas disponíveis para avaliar essa suposição. $O$ câncer de estômago costuma estar associado, também, a baixas condições sócio-econômicas.

Não se conhece o comportamento do câncer de estômago no Brasil. São muito poucos os dados sobre a incidência dessa doença, pois existem em funcionamento, atualmente, apenas cinco Registros de Câncer de Base Populacional (Porto Alegre, Fortaleza, Belém, Goiania e Campinas), sendo que as séries históricas disponíveis são pequenas para se avaliarem tendências com algum grau de confiabilidade.

No entanto, há séries históricas de mortalidade para o país inteiro, padronizadas, a partir de 1977, possibilitando a avaliação do comportamento de qualquer causa básica de óbito. Vale lembrar que descrever o comportamento de um fenômeno através do tempo e verificar a existência de tendências estão entre os principais objetivos da análise de séries históricas. Particularmente, neste estudo, tem-se o interesse em avaliar como o câncer de estômago está se comportando em nosso meio.

Este trabalho analisa a tendência da mortalidade por câncer de estômago no Brasil e nas capitais brasileiras, para o período de 1977 a 1989.

Material e métodos

Óbitos

Utilizou-se a série histórica de óbitos para os anos de 1977 a 1989, período em que estão disponíveis os dados de mortalidade fornecidos pelo Ministério da Saúde (MS, 1979-1993), com exceção de Vitória, cuja série histórica iniciouse em 1978, por não se dispor dos dados de 1977.

Optou-se por trabalhar com o Brasil como um todo e apenas com as capitais brasileiras devido à melhor qualidade do preenchimento da Declaração de óbito (DO) e à maior cobertura do Sistema de Mortalidade do Ministério da Saúde nesses locais (Mello Jorge \& Latorre, 1994). 
Número, mortalidade proporcional (\%)* (MP) e o posto** na mortalidade geral dos óbitos por neoplasias malignas.*** Brasil e capitais, 1978 e 1989.

\begin{tabular}{|c|c|c|c|c|c|c|}
\hline local & $\mathrm{n}$ & $\begin{array}{c}1978 \\
M P *(\%)\end{array}$ & Posto** & $\mathrm{n}$ & $\begin{array}{c}1989 \\
M P^{*}(\%)\end{array}$ & Posto** \\
\hline Brasil & 54.840 & 8,3 & 5으 & 80.614 & 9,9 & 30 \\
\hline \multicolumn{7}{|l|}{ Região Norte } \\
\hline Rio Branco & 46 & 6,5 & $5 \underline{0}$ & 76 & 6,5 & $5 \underline{0}$ \\
\hline Manaus & 325 & 7,5 & $5 \underline{0}$ & 487 & 8,2 & $5 \underline{0}$ \\
\hline Boa Vista & 26 & 8,3 & 5으 & 40 & 5,7 & 5으 \\
\hline Belém & 665 & 9,6 & $4 \underline{0}$ & 763 & 13,2 & $2 \underline{0}$ \\
\hline Macapá & 59 & 9,4 & $6 \underline{0}$ & 108 & 14,6 & $2 \underline{0}$ \\
\hline Porto Velho & 34 & 3,5 & 7으 & 110 & 6,6 & $5 \underline{0}$ \\
\hline \multicolumn{7}{|l|}{ Região Nordeste } \\
\hline São Luís & 178 & 6,4 & 5으 & 308 & 9,4 & $4 \underline{0}$ \\
\hline Teresina & 132 & 5,2 & $6 \underline{0}$ & 304 & 10,9 & $3 \underline{0}$ \\
\hline Fortaleza & 642 & 6,6 & 5으 & 1140 & 12,3 & $2 \underline{0}$ \\
\hline N atal & 236 & 8,2 & $4 \underline{0}$ & 392 & 13,6 & $2 \underline{0}$ \\
\hline J oão Pessoa & 179 & 6,0 & 5으 & 264 & 8,0 & $4 \underline{0}$ \\
\hline Recife & 852 & 8,6 & $4 \underline{0}$ & 1.039 & 10,3 & 30 \\
\hline Salvador & 858 & 7,3 & $6 \underline{0}$ & 1.290 & 11,2 & $4 \underline{0}$ \\
\hline Maceió & 201 & 4,9 & $6 \underline{0}$ & 334 & 8,2 & 5으 \\
\hline Aracaju & 105 & 4,3 & $7 \underline{0}$ & 267 & 9,1 & 5으 \\
\hline \multicolumn{7}{|l|}{ Região Sudeste } \\
\hline Belo Horizonte & 1.204 & 9,1 & 5으 & 1.623 & 12,6 & $2 \underline{0}$ \\
\hline Vitória & 99 & 9,0 & $6 \underline{0}$ & 177 & 12,4 & 30 \\
\hline Rio de J aneiro & 5.352 & 13,6 & $2 \underline{0}$ & 6.407 & 13,3 & 3으 \\
\hline São Paulo & 6.660 & 11,8 & $4 \underline{0}$ & 8.407 & 13,3 & 3으 \\
\hline \multicolumn{7}{|l|}{ Região Sul } \\
\hline Curitiba & 822 & 12,0 & $4 \underline{0}$ & 1.209 & 14,9 & $2 \underline{0}$ \\
\hline Florianópolis & 138 & 11,9 & $3 \underline{0}$ & 216 & 16,4 & $2 \underline{0}$ \\
\hline Porto Alegre & 1.115 & 14,8 & $2 \underline{0}$ & 1.654 & 18,2 & $2 \underline{0}$ \\
\hline \multicolumn{7}{|c|}{ Região Centro-O este } \\
\hline Campo Grande & 159 & 7,5 & 5으 & 3.339 & 12,3 & 3으 \\
\hline Cuiabá & 80 & 5,5 & $4 \underline{0}$ & 172 & 9,9 & 3으 \\
\hline Goiânia & 307 & 7,2 & $6 \underline{0}$ & 497 & 10,4 & 3으 \\
\hline Brasília & 451 & 8,5 & $6 \underline{0}$ & 809 & 12,9 & 3으 \\
\hline
\end{tabular}

* $\mathrm{MP}=$ porcentagem de óbito por neoplasias malignas dentre o total de óbitos.

** posto: posição na mortalidade geral, considerando todos os capítulos.

*** neoplasias malignas: capítulo II da CID 9ạ revisão.

População

Estimou-se a população para 1o de julho, referente aos anos de estudo, por meio de interpolação (método de Lagrange) com base nos dados dos Censos de 1970 (IBGE, 1973), 1980 (IBGE, 1982) e 1991 (IBGE, dados não publicados).

\section{Mortalidade proporcional}

Calculou-se a mortalidade proporcional (MP) de câncer de estômago (categoria 151 da CID 9ạ revisão), dentro de todos os óbitos por neoplasias (capítulo II da CID 9ạ revisão), para avaliar a importância dessa localização de câncer em relação a todos os cânceres. Nessa situação, a MP é calculada dividindo-se os óbitos por câncer de estômago pelo total de óbitos por neoplasias. Também foi calculada a MP por neoplasias dentre o total de óbitos. 
Coeficientes de mortalidade

(por cem mil habitantes)

Foram calculados o coeficiente de mortalidade bruto por câncer de estômago (número de óbitos por essa localização dividido pela população do meio do ano) e o coeficiente de mortalidade padronizado (utilizando-se como população padrão a do Brasil, para 1o de setembro de 1980), tendo como base cem mil habitantes.

\section{A nálise da tendência}

A análise da tendência foi feita com os coeficientes padronizados de mortalidade por câncer de estômago.

Inicial mente, foram feitos os diagramas de dispersão entre os coeficientes de mortalidade padronizados e os anos de estudo, para se visualizar o tipo de relacionamento entre eles.

A seguir, foi iniciado o processo de modelagem, considerando os coeficientes padronizados de mortalidade por câncer de estômago como variável dependente $(Y)$ e os anos de estudo como variável independente (X). Para o estudo da tendência, optou-se por estimar modelos de regressão (Boyle \& Parkin, 1991; Kleimbaum et al., 1988; Moretin \& Toloi, 1986). A vantagem em se utilizar essa classe de modelos estatísticos para análise de séries temporais é a facilidade de sua elaboração e interpretação, além de serem mais poderosos do ponto de vista estatístico. Para se evitar a colinearidade entre os termos da equação de regressão, utilizou-se a variável centralizada (ou seja, ao invés de se trabalhar com $X$, utilizou-se o termo (X-1983), pois 1983 é o ponto médio da série histórica).

O primeiro modelo a ser testado foi o de regressão linear simples $\left(Y=\beta_{0}+\beta_{1} X\right)$ e, posteriormente, foram testados os modelos de ordem maior: segundo grau ou parabólico $\left(Y=\beta_{0}\right.$ $\left.+\beta_{1} X+\beta_{2} X^{2}\right)$ terceiro grau $\left(Y=\beta_{0}+\beta_{1} X+\beta_{2} X^{2}+\right.$ $\beta_{3} x^{3}$ ) e exponencial $\left(Y=e \beta_{0}+\beta_{1} x\right)$.

Considerou-se como melhor modelo aquele que apresentou maior significância estatística (menor valor de $\mathrm{p}$ ) e resíduos sem vícios. Quando dois modelos foram semelhantes, do ponto de vista estatístico, para a mesma localidade, optou-se pelo model o mais simples, ou seja, de menor ordem. Considerou-se tendência significante aquela cujo modelo estimado obteve $p \triangleleft 0,05$.

\section{Resultados}

A Tabela 2 apresenta o número de óbitos por câncer do estômago, a mortalidade proporcional dessa localização dentre as neoplasias, os coeficientes de mortalidade brutos (CB) e padronizados (CP) para 1978 e 1989 e a mudança ocorrida entre esses anos no coeficiente padronizado $\left[\left(\mathrm{CP}_{1978}-\mathrm{CP}_{1989}\right) / \mathrm{CP}_{1978}\right]$ para o Brasil e suas capitais.

Observa-se que o coeficiente de mortalidade por câncer de estômago (padronizado), para o Brasil, reduziu-se em 50\%, passando de 10,4 em 1978, para 5,2 por cem mil habitantes em 1989. Para esse mesmo período, o coeficiente de mortalidade por câncer, para o Brasil, passou de 48,4 para 56,7 por cem mil habitantes (aumento de 17\%), sendo que, atualmente, o pulmão éa localização mais freqüente dentre os óbitos por câncer para o sexo masculino, e a mama dentre as mulheres, passando o estômago para segundo lugar, em ambos os sexos.

Na Região Norte, em grande parte das capitais, os coeficientes padronizados de mortalidade por câncer de estômago de 1989 representam cerca de 30\% daqueles referentes a 1978. O maior decréscimo ocorreu em Boa Vista $(93,7 \%)$ e o menor em Macapá $(41,5 \%)$. Vale salientar que, devido ao pequeno número de óbitos em algumas capitais, houve uma oscilação muito grande nos coeficientes, prejudicando a comparação. Analisando as mortalidades proporcionais, tem-se uma redução de importância do câncer de estômago dentre o total de neoplasias em todas as capitais da Região Norte, com exceção de Porto Velho, que passou de $14,7 \%$ para 16,4\%, com declínio, porém, tanto do coeficiente de mortalidade bruto, quanto do padronizado. No entanto, nessas capitais, o câncer de estômago ainda é a localização mais freqüente dentre os homens, com exceção de Manaus (segundo lugar) e Boa Vista (oitavo lugar). Nas mulheres, o câncer de estômago é a segunda ou terceira causa dentre todos os óbitos por câncer, exceto, novamente, em Boa Vista, onde o estômago ocupa a oitava posição, também entre as mulheres. Nelas, o útero e, às vezes, a mama são mais freqüentes.

Já na Região Nordeste, os decréscimos observados nos coeficientes de mortalidade por câncer de estômago padronizados são mais expressivos, exceto para Aracaju, onde o coeficiente referente ao ano de 1989 é 1,14 vezes o de 1978, embora a mortalidade proporcional tenha permanecido a mesma $(6,7 \%$ dentre os neoplasmas). No restante das capitais, o declínio nos coeficientes variou de $28,7 \%$ (Teresina) a 58,6\% (Salvador). O câncer de estômago per- 
Número, mortalidade proporcional por câncer de estômago dentre as neoplasias (MP),

coeficientes de mortalidade bruto*(CB) e padronizado** (CP) por câncer de estômago. Brasil e capitais, 1978 e 1989.

\begin{tabular}{|c|c|c|c|c|c|c|c|c|c|}
\hline \multirow{2}{*}{ local } & \multicolumn{4}{|c|}{1978} & \multicolumn{4}{|c|}{1989} & \multirow{2}{*}{$\begin{array}{c}\text { comparação*** } \\
(\%)\end{array}$} \\
\hline & $\mathrm{n}$ & MP (\%) & $\mathrm{CB}^{*}$ & $\mathrm{CP} * *$ & $\mathrm{n}$ & MP (\%) & $\mathrm{CB}^{*}$ & $\mathrm{CP} * *$ & \\
\hline Brasil & 8.265 & 15,1 & 7,3 & 10,4 & 9.569 & 11,9 & 6,7 & 5,2 & 50,0 \\
\hline \multicolumn{10}{|l|}{ Região Norte } \\
\hline Rio Branco & 8 & 17,4 & 8,0 & 16,3 & 11 & 14,5 & 6,3 & 4,5 & 72,4 \\
\hline Manaus & 77 & 23,7 & 13,8 & 28,0 & 66 & 13,6 & 7,1 & 9,3 & 66,8 \\
\hline Boa Vista & 5 & 19,2 & 6,6 & 19,0 & 1 & 2,5 & 0,8 & 1,2 & 93,7 \\
\hline Belém & 149 & 22,4 & 17,2 & 27,8 & 118 & 15,5 & 10,0 & 10,1 & 63,7 \\
\hline Macapá & 18 & 30,5 & 14,1 & 33,0 & 27 & 25,0 & 15,7 & 19,3 & 41,5 \\
\hline Porto Velho & 5 & 14,7 & 8,0 & 16,3 & 18 & 16,4 & 6,3 & 4,5 & 72,4 \\
\hline \multicolumn{10}{|l|}{ Região Nordeste } \\
\hline São Luís & 25 & 14,0 & 6,2 & 10,6 & 40 & 13,0 & 6,2 & 6,8 & 35,8 \\
\hline Teresina & 19 & 14,4 & 5,6 & 10,8 & 18 & 5,9 & 7,3 & 7,7 & 28,7 \\
\hline Fortaleza & 117 & 18,2 & 9,7 & 14,3 & 170 & 14,9 & 10,1 & 9,1 & 36,4 \\
\hline Natal & 33 & 14,0 & 9,6 & 13,6 & 52 & 13,3 & 9,4 & 7,5 & 44,9 \\
\hline J oão Pessoa & 12 & 6,7 & 4,0 & 5,0 & 15 & 5,7 & 3,3 & 2,1 & 58,0 \\
\hline Recife & 83 & 9,7 & 7,1 & 9,3 & 88 & 8,5 & 6,9 & 4,0 & 57,0 \\
\hline Salvador & 105 & 12,2 & 7,6 & 12,8 & 104 & 8,1 & 5,3 & 5,3 & 58,6 \\
\hline Maceió & 16 & 8,0 & 4,7 & 7,1 & 18 & 5,4 & 3,2 & 3,1 & 56,3 \\
\hline Aracaju & 7 & 6,7 & 2,6 & 4,2 & 18 & 6,7 & 4,7 & 4,8 & $-14,3$ \\
\hline \multicolumn{10}{|l|}{ Região Sudeste } \\
\hline Belo Horizonte & 153 & 12,7 & 9,1 & 9,2 & 176 & 10,8 & 8,7 & 4,2 & 54,3 \\
\hline Vitória & 20 & 20,2 & 10,3 & 14,8 & 14 & 7,9 & 5,6 & 7,3 & 50,7 \\
\hline Rio de J aneiro & 672 & 12,6 & 13,6 & 13,3 & 590 & 9,2 & 10,8 & 4,3 & 67,7 \\
\hline São Paulo & 937 & 14,1 & 11,7 & 15,0 & 974 & 11,6 & 10,2 & 8,4 & 44,0 \\
\hline \multicolumn{10}{|l|}{ Região Sul } \\
\hline Curitiba & 117 & 14,2 & 12,4 & 18,6 & 132 & 10,9 & 10,4 & 8,5 & 54,3 \\
\hline Florianópolis & 15 & 10,9 & 7,7 & 11,2 & 19 & 8,8 & 8,3 & 6,7 & 40,2 \\
\hline Porto Alegre & 74 & 6,6 & 6,8 & 7,9 & 98 & 5,9 & 7,9 & 4,9 & 38,0 \\
\hline \multicolumn{10}{|c|}{ Região Centro-O este } \\
\hline Campo Grande & 19 & 12,0 & 7,6 & 13,5 & 42 & 12,4 & 8,9 & 8,5 & 37,0 \\
\hline Cuiabá & 9 & 11,3 & 4,9 & 10,0 & 14 & 8,1 & 3,9 & 4,4 & 56,0 \\
\hline Goiânia & 38 & 12,4 & 5,8 & 12,5 & 46 & 9,3 & 5,1 & 5,4 & 56,8 \\
\hline Brasília & 38 & 8,4 & 3,7 & 10,0 & 68 & 8,4 & 4,3 & 5,8 & 42,0 \\
\hline
\end{tabular}

* CB: coeficiente de mortalidade por câncer de estômago (por cem mil habitantes).

** CP: coeficiente padronizado de mortalidade por câncer de estômago (por cem mil habitantes).

*** Comparação: $\left(\mathrm{CP}_{78}-\mathrm{CP}_{89}\right) / \mathrm{CP}_{78}$

de a importância dentre os óbitos por neoplasmas, pois, comparando-se os anos de 1978 e 1989, a mortalidade proporcional diminuiu em todas as capitais do Nordeste, com exceção de Aracaju, onde a mortalidade proporcional se manteve em 6,7\%. João Pessoa, Maceió e Recife apresentaram os menores coeficientes de mortalidade padronizados (respectivamente: 2,1 ; 3,1 e 4,0 por cem mil habitantes), abaixo da média nacional (5,2 por cem mil habitantes).
Dentre os homens, o câncer de estômago ainda é a localização mais importante em São Luís, Teresina, Fortaleza, João Pessoa, Natal e Salvador, dando lugar ao pulmão em Recife e Maceió e ocupando quarto posto em Aracaju e o sexto em João Pessoa. Para as mulheres, o comportamento é semelhante ao da Região Norte, estando sempre em segundo ou terceiro lugar, excetuando-se Maceió e Aracaju (quarto lugar) e João Pessoa (sexto lugar). 
A região Sudeste também apresentou queda nos coeficentes de mortalidade por câncer de estômago padronizados, sendo o maior declínio no Rio de Janeiro e o menor em São Paulo (67,7\% e 44,0\%). Esse declínio refletiu-se na importância dessa localização dentre os neoplasmas, onde todos caíram de dois a três pontos percentuais na mortalidade proporcional, excluindo Vitória, que, de 20,2\%, o câncer de estômago passou para 7,9\% do total dos cânceres. Vale salientar que tanto Belo Horizonte, quanto Rio de Janeiro apresentaram coeficientes de mortalidade padronizados bem baixos $(4,2$ e 4,3 por cem mil habitantes, respectivamente), praticamente metade das outras capitais da Região Sudeste e abaixo da média nacional. Em qualquer das capitais desta região, o câncer de estômago ocupa sempre a segunda posição na mortalidade por neoplasias, em ambos os sexos, cedendo lugar para o pulmão (sexo masculino) e mama (sexo feminino), exceto em Vitória, onde é a quinta localização mais freqüente de câncer dentre as mulheres (útero, mama, pulmão e cólon são mais comuns).

O comportamento dos coeficientes padronizados de mortalidade por câncer de estômago nas capitais da Região Sul é semelhante ao observado no Sudeste. Curitiba, Florianópolis e Porto Alegre tiveram redução nos coeficientes (respectivamente: $54,3 \% ; 40,2 \%$ e $38,0 \%$ ) e perda de importância dentre o total de neoplasias, principalmente em Curitiba (de 14,2\% em 1978, passou para 10,9\% em 1989). No entanto, o menor coeficiente padronizado encontra-se em Porto Alegre (4,9 por cem mil habitantes). É a segunda causa de óbito por câncer entre os homens em Curitiba e Florianópolis (pulmão é a primeira) e quarta em Porto Alegre (pulmão, próstata e esôfago, na frente). Em relação às mulheres, ocupa a quarta posição em Curitiba (mama, útero e pulmão, primeiro) equinta em Florianópolis (mama, útero, pâncreas e cólon, antes ) e Porto Alegre (mama, útero, pulmão e cólon vêm primeiro).

Da mesma forma, na Região Centro-Oeste, verifica-se um decréscimo considerável nos coeficientes padronizados de mortalidade por câncer de estômago, sendo o maior deles verificado em Goiânia e o menor em Campo Grande (respectivamente, 56,8\% e 37,0\% em relação aos coeficientes de 1978). Entretanto, a mortalidade proporcional dentre o total de neoplasias aumentou um pouco em Campo Grande (de $12,0 \%$ em 1978 passou para $12,4 \%$ em 1989 ), estacionou em Brasília (8,4\%) e declinou nas duas outras capitais em, praticamente, três pontos percentuais, mostrando ter diminuído a importância do câncer de estômago como causa bási- ca de óbito. O menor coeficiente padronizado encontra-se em Cuiabá (4,4 por cem mil habitantes) e o maior em Campo Grande (8,5 por cem mil habitantes), confirmando a importância dessa localização de câncer nesta última capital. Em relação ao sexo masculino, apenas em Campo Grande o óbito por câncer de estômago é a categoria mais freqüente de neoplasmas, ocupando o segundo posto nas outras capitais do Centro-Oeste (pulmão é o primeiro). Já no sexo feminino, o útero e a mama são mais importantes em Campo Grande e em Brasília; a essas duas local izações junta-se o pulmão em Cuiabá e em Goiânia, ocupando, o estômago, o quarto posto nessas duas últimas capitais.

Verificou-se, portanto, que, praticamente em todas as capitais brasileiras e no Brasil como um todo, houve um decréscimo importante nos coeficientes padronizados de mortalidade por câncer de estômago, indicando que a intensidade com que essa causa de óbito ocorre nas populações estudadas diminuiu quando se comparam 1978 e 1989.

Embora o câncer de estômago ainda seja uma causa de óbito importante dentre os neoplasmas, principalmente em algumas capitais das Regiões Norte e Nordeste, a porcentagem desta localização de câncer vem declinando nas Regiões Sudeste, Sul e Centro-Oeste, tendo a mortalidade proporcional dentre os neoplasmas diminuído na maioria das capitais.

As Tabelas 3 e 4 apresentam os resultados da análise das tendências dos coeficientes padronizados de mortalidade por câncer de estômago para os sexos masculino efeminino, respectivamente.

O modelo que melhor representa o comportamento da tendência da mortalidade por câncer de estômago para o Brasil , para ambos os sexos, é o de terceira ordem. Este revela uma tendência decrescente não constante, durante boa parte do período, tanto para os homens, quanto para as mulheres, e, em anos mais recentes, houve uma estabilização dos coeficientes padronizados. Se o objetivo deste trabalho fosse estimar os valores dos coeficientes de mortalidade para os próximos anos, o modelo de terceiro grau não seria o mais adequado, pois forneceria valores crescentes dos coeficientes a partir de 1989, fato não esperado. Para projeções, o melhor modelo é o linear, porém, como se deseja avaliar a tendência da última década, o modelo de terceira ordem é o mais significante do ponto de vista estatístico ( $p \varangle 0,001$ ), para ambos os sexos. Optou-se, por isso, por selecioná-lo para esta análise.

Na Região Norte, conseguiu-se definir tendências significantes em Rio Branco, Manaus e 
Resultados da análise de tendência dos coeficientes padronizados de mortalidade por câncer de estômago para o sexo masculino. Brasil e capitais, 1977 a 1989.

\begin{tabular}{|c|c|c|c|c|}
\hline local & modelo* & $\mathrm{R}^{2}(\%)$ & $p$ & tendência \\
\hline Brasil & $y=11,17-1,16 x-0,02 x^{2}+0,02 x^{3}$ & 91,1 & $<0,001$ & $\begin{array}{l}\text { Decrescente a maior parte do período, } \\
\text { porém estável ao final }\end{array}$ \\
\hline \multicolumn{5}{|l|}{ Região Norte } \\
\hline Rio Branco & $y=14,30-1,80 x$ & 52,0 & 0,005 & Decrescente e constante \\
\hline Manaus & $y=25,93-2,31 x$ & 88,9 & $<0,001$ & Decrescente e constante \\
\hline Boa Vista & $y=10,34-1,06 x$ & 24,6 & 0,085 & Não significante \\
\hline Belém & $y=27,86-2,19 x$ & 74,8 & $<0,001$ & Decrescente e constante \\
\hline Macapá & $y=28,80+1,66 x+0,17 x^{2}-0,06 x^{3}$ & 7,4 & 0,867 & Não significante \\
\hline Porto Velho & $y=15,86-0,46 x-0,14 x^{2}+0,03 x^{3}$ & 47,7 & 0,106 & Não significante \\
\hline \multicolumn{5}{|l|}{ Região Nordeste } \\
\hline São Luís & $y=14,39-0,18 x-0,13 x^{2}$ & 18,5 & 0,360 & Não significante \\
\hline Teresina & $y=6,00-0,45 x+0,14 x^{2}$ & 33,7 & 0,128 & Não significante \\
\hline Fortaleza & $y=16,00-1,77 x-0,06 x^{2}+0,03 x^{3}$ & 87,9 & $<0,001$ & $\begin{array}{l}\text { Decrescente a maior parte do período, } \\
\text { porém estável ao final }\end{array}$ \\
\hline Natal & $y=15,43-1,00 x$ & 60,3 & 0,002 & Decrescente e constante \\
\hline J oão Pessoa & $y=9,71-1,22 x-0,13 x^{2}+0,03 x^{3}$ & 70,5 & 0,009 & $\begin{array}{l}\text { Decrescente a maior parte do período, } \\
\text { porém estável ao final }\end{array}$ \\
\hline Recife & $y=8,63-0,85 x$ & 74,7 & $<0,001$ & Decrescente e constante \\
\hline Salvador & $y=11,78-0,70 x$ & 61,5 & 0,002 & Decrescente e constante \\
\hline Maceió & $y=9,32-0,86 x$ & 54,2 & 0,004 & Decrescente e constante \\
\hline Aracaju & $y=8,53-0,65 x$ & 37,8 & 0,025 & Decrescente e constante \\
\hline \multicolumn{5}{|l|}{ Região Sudeste } \\
\hline Belo Horizonte & $y=15,43-0,58 x-0,16 x^{2}$ & 68,8 & 0,003 & Decrescente, não constante \\
\hline Vitória & $y=18,53-0,81 z(* *)$ & 75,8 & $<0,001$ & Decrescente e constante \\
\hline Rio de J aneiro & $y=14,19-1,30 x$ & 34,2 & 0,036 & Decrescente e constante \\
\hline São Paulo & $y=15,66-1,82 x+0,03 x^{2}+0,03 x^{3}$ & 88,8 & $<0,001$ & $\begin{array}{l}\text { Decrescente a maior parte do período, } \\
\text { porém estável ao final }\end{array}$ \\
\hline \multicolumn{5}{|l|}{ Região Sul } \\
\hline Curitiba & $y=19,04-1,51 x$ & 79,6 & $<0,001$ & Decrescente e constante \\
\hline Florianópolis & $y=17,37-0,87 x$ & 47,1 & 0,010 & Decrescente e constante \\
\hline Porto Alegre & $y=11,27-0,77 x$ & 68,8 & $<0,001$ & Decrescente e constante \\
\hline \multicolumn{5}{|c|}{ Região Centro-Oeste } \\
\hline Campo Grande & $y=14,81-1,19 x-0,14 x^{2}+0,05 x^{3}$ & 40,6 & 0,177 & Não significante \\
\hline Cuiabá & $y=15,60-0,43 x-0,25 x^{2}$ & 73,4 & 0,001 & Decrescente, não constante \\
\hline Goiânia & $y=13,12-2,75 x+0,06 x^{3}$ & 73,1 & 0,001 & $\begin{array}{l}\text { Decrescente a maior parte do período, } \\
\text { porém estável ao final }\end{array}$ \\
\hline Brasília & $y=15,38-1,19 x-0,16 x^{2}+0,05 x^{3}$ & 80,9 & 0,001 & $\begin{array}{l}\text { Decrescente a maior parte do período, } \\
\text { porém estável ao final }\end{array}$ \\
\hline
\end{tabular}

* Modelo:

$y=$ coeficiente de mortalidade padronizado de câncer de estômago (por cem mil hab.); $x=$ ano - 1983

** para Vitória tem-se que $z=($ ano $-1983,5) x$

Belém. Nestas cidades, houve tendência decrescente e constante (modelo linear) no coeficiente de mortalidade por câncer de estômago padronizado, tanto para o sexo masculino, quanto para o feminino, porém para este último, em Rio Branco, esta tendência não foi constante (modelo de terceiro grau) e, nos últi- mos anos, a tendência é de estabilidade, muito provavel mente pelo pequeno número de óbitos, que provoca uma oscilação muito grande nos coeficientes. Este mesmo fenômeno prejudicou o ajuste de um modelo significante aos dados das outras capitais da Região Norte (Boa Vista, Macapá e Porto Velho), embora possa ser 
Tabela 4

Resultados da análise de tendência dos coeficientes padronizados de mortalidade por câncer de estômago para o sexo feminino. Brasil e capitais, 1977 a 1989.

\begin{tabular}{|c|c|c|c|c|}
\hline local & modelo* & $\mathrm{R}^{2}(\%)$ & $\mathrm{p}$ & tendência \\
\hline Brasil & $y=5,24-0,70 x+0,01 x^{3}$ & 94,4 & $<0,001$ & $\begin{array}{l}\text { Decrescente a maior parte do período, } \\
\text { porém estável ao final }\end{array}$ \\
\hline \multicolumn{5}{|l|}{ Região Norte } \\
\hline Rio Branco & $y=8,01-2,40 x-0,06 x^{3}$ & 53,1 & 0,023 & $\begin{array}{l}\text { Decrescente a maior parte do período, } \\
\text { porém estável ao final }\end{array}$ \\
\hline Manaus & $y=11,63-0,96 x$ & 56,4 & 0,003 & Decrescente e constante \\
\hline Boa Vista & $y=2,72-0,94 x-0,04 x^{3}$ & 38,1 & 0,090 & Não significante \\
\hline Belém & $y=10,73-0,80 x$ & 72,2 & $<0,001$ & Decrescente e constante \\
\hline Macapá & $y=12,39-0,57 x$ & 17,6 & 0,153 & Não significante \\
\hline Porto Velho & $y=6,15+0,38 x$ & 11,4 & 0,259 & Não significante \\
\hline \multicolumn{5}{|l|}{ Região Nordeste } \\
\hline São Luís & $y=5,99-0,45 x$ & 68,0 & $<0,001$ & Decrescente e constante \\
\hline Teresina & $y=3,59-0,43 x+0,02 x^{2}$ & 37,7 & 0,094 & Não significante \\
\hline Fortaleza & $y=7,55-1,07 x+0,03 x^{3}$ & 59,8 & 0,010 & $\begin{array}{l}\text { Decrescente a maior parte do período, } \\
\text { porém estável ao final }\end{array}$ \\
\hline Natal & $y=7,22-1,17 x+0,03 x^{3}$ & 54,1 & 0,020 & $\begin{array}{l}\text { Decrescente a maior parte do período, } \\
\text { porém estável ao final }\end{array}$ \\
\hline J oão Pessoa & $y=3,00-0,32 x$ & 56,5 & 0,003 & Decrescente e constante \\
\hline Recife & $y=4,45-0,28 x$ & 44,1 & 0,013 & Decrescente e constante \\
\hline Salvador & $y=6,68-0,58 x$ & 77,4 & $<0,001$ & Decrescente e constante \\
\hline Maceió & $y=3,46-0,20 x$ & 24,0 & 0,090 & Não significante \\
\hline Aracaju & $y=6,00-1,32 x-0,09 x^{2}+0,04 x^{3}$ & 66,9 & 0,015 & $\begin{array}{l}\text { Decrescente a maior parte do período, } \\
\text { porém estável ao final }\end{array}$ \\
\hline \multicolumn{5}{|l|}{ Região Sudeste } \\
\hline Belo Horizonte & $y=7,60-0,28 x-0,07 x^{2}$ & 66,1 & 0,005 & Decrescente, não constante \\
\hline Vitória & $y=8,56-0,23 z-0,03 z^{2}\left(^{* *}\right)$ & 50,8 & 0,041 & Decrescente, não constante \\
\hline Rio de J aneiro & $y=7,42-0,76 x$ & 62,8 & 0,001 & Decrescente e constante \\
\hline São Paulo & $y=7,47-0,95 x+0,02 x^{2}+0,01 x^{3}$ & 92,9 & $<0,001$ & $\begin{array}{l}\text { Decrescente a maior parte do período, } \\
\text { porém estável ao final }\end{array}$ \\
\hline \multicolumn{5}{|l|}{ Região Sul } \\
\hline Curitiba & $y=8,26-0,80 x$ & 80,9 & $<0,001$ & Decrescente e constante \\
\hline Florianópolis & $y=7,43-1,12 x-0,06 x^{2}+0,04 x^{3}$ & 23,1 & 0,478 & Não significante \\
\hline Porto Alegre & $y=6,15-0,25 x-0,05 x^{2}$ & 61,0 & 0,009 & Decrescente, não constante \\
\hline \multicolumn{5}{|c|}{ Região Centro-Oeste } \\
\hline Campo Grande & $y=6,79-1,40 x+0,04 x^{3}$ & 39,0 & 0,085 & Não significante \\
\hline Cuiabá & $y=5,05-1,14 x-0,03 x^{3}$ & 32,5 & 0,140 & Não significante \\
\hline Goiânia & $y=6,80-1,21 x-0,08 x^{2}+0,04 x^{3}$ & 57,4 & 0,045 & $\begin{array}{l}\text { Decrescente a maior parte do período, } \\
\text { porém estável ao final }\end{array}$ \\
\hline Brasília & $y=7,63-1,03 x-0,05 x^{2}+0,02 x^{3}$ & 74,9 & 0,005 & $\begin{array}{l}\text { Decrescente a maior parte do período, } \\
\text { porém estável ao final }\end{array}$ \\
\hline
\end{tabular}

* Modelo:

$y=$ coeficiente de mortalidade padronizado de câncer de estômago (por cem mil hab.); $x=$ ano - 1983

** para Vitória tem-se que $\mathrm{z}=($ ano $-1983,5) \mathrm{x}$ 
visualizada uma tendência de declínio, com grande oscilação dos dados, durante o período considerado, em ambos os sexos.

Padrão semelhante se observa nas capitais do Nordeste, pois grande parte dessas capitais apresentou tendência de decréscimo nos coeficientes padronizados para ambos os sexos. Analisando os homens, encontrou-se que, para Natal, Recife, Salvador, Maceió e Aracaju, essa tendência foi constante e estatisticamente significante, e não constante em Fortaleza e João Pessoa. Para o sexo feminino, a tendência é de declínio e constante em São Luís, João Pessoa, Recife e Salvador. Em Fortaleza, Natal, e Aracaju, o melhor modelo foi o de terceiro grau, mostrando um tendência de decréscimo nos coeficientes padronizados de mortalidade por câncer de estômago, porém essa tendência não apresenta velocidade constante em todo o período. A análise de Teresina ficou muito prejudicada pela grande oscilação dos valores dos coeficientes padronizados.

Todos os modelos ajustados para as capitais da Região Sudeste foram estatisticamente significantes, apresentando tendências de declínio nos coeficientes padronizados de mortalidade por câncer de estômago, tanto para os homens quanto para as mulheres. Os padrões desses declínios foram diferentes, e apenas no Rio de Janeiro (ambos os sexos) e em Vitória (sexo masculino) essa tendência foi constante no período de estudo.

No Sul, para o sexo masculino, em todas as capitais, a tendência foi de decréscimo constante da mortalidade por câncer de estômago, no período de 1977 a 1989, sempre estatisticamente significante. Já no sexo feminino, verifica-se que o padrão constante manteve-se apenas em Curitiba. Entre as mulheres, em Florianópolis, não se consegue definir uma tendência estatisticamente significante, provavelmente pelo pequeno número de óbitos. Em Porto Alegre, observou-se uma tendência de decréscimo do coeficiente de mortalidade por câncer de estômago dentre as mulheres, porém não constante.

A maioria das capitais do Centro-Oeste apresenta tendência de declínio não constante nos coeficientes padronizados de mortalidade por câncer de estômago, exceto em Campo Grande, onde há grande oscilação nos coeficientes, não se detectando qualquer tendência estatisticamente significante em ambos os sexos.

As Figuras 1 e 2 resumem as tendências dos coeficientes padronizados de mortalidade por câncer de estômago para o Brasil e suas capitais.
Figura 1

Tendências da mortalidade por câncer de estômago. Capitais brasileiras, sexo masculino, 1977 a 1989.

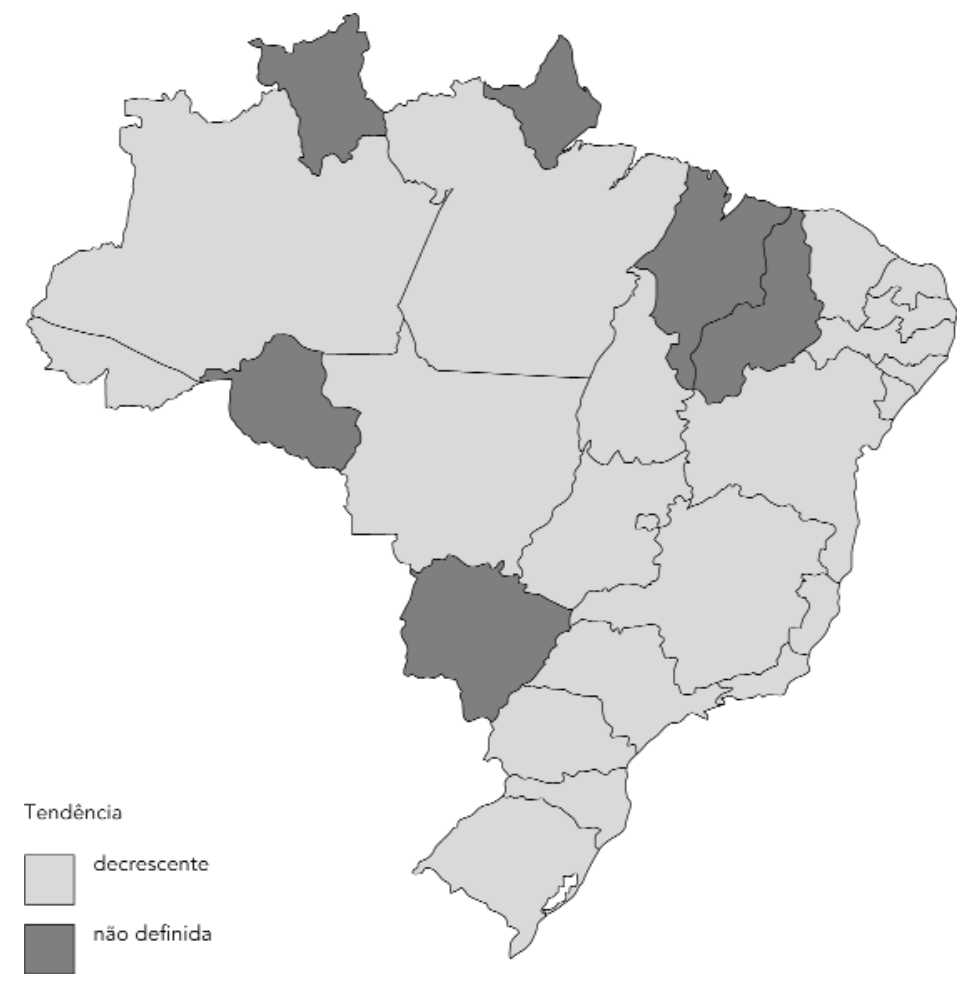

Discussão

Embora a mortalidade não quantifique a incidência de uma doença, uma vez que a letalidade do câncer de estômago não tem se alterado nos últimos anos, pode-se pensar que as tendências encontradas refletiriam a tendência da incidência do câncer do estômago em nosso meio. Isso também vale para outras partes do mundo (Coleman et al., 1993). Porém, somente com a comparação entre as tendências da incidência e da mortalidade poderia ser avaliado corretamente esse fenômeno. Se ambas estivessem diminuindo, isso seria uma demonstração de que a verdadeira incidência da doença estaria decrescendo, não sendo, pois, decorrente de mudanças em técnicas de diagnóstico (NCIC, 1992; McCredie et al., 1992).

Em recente trabalho em que analisam as tendências da incidência e da mortalidade para diversos tipos de câncer em várias localidades, Coleman et al. (1993) afirmam que a incidência de câncer de estômago na Europa diminuiu em 22 das 23 populações estudadas, com taxas de $10 \%$ a $30 \%$ para cada cinco anos, em ambos os sexos, o mesmo ocorrendo com a 
Figura 2

Tendências da mortalidade por câncer de estômago. Capitais brasileiras, sexo feminino, 1977 a 1989.

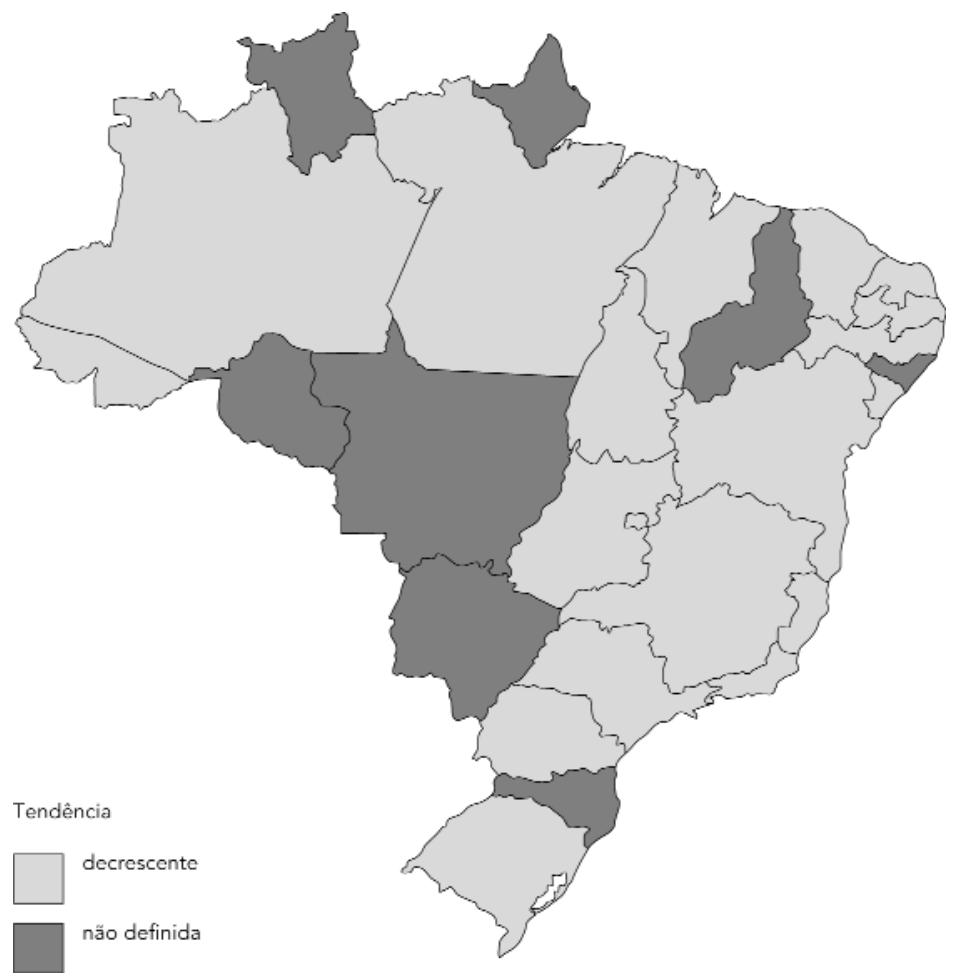

mortalidade. Mesmo países europeus com altas taxas de incidência, como Portugal, Hungria, Polônia, Romênia, apesar do decréscimo mais lento, também vêm diminuindo suas taxas tanto de incidência, quanto de mortalidade. A incidência também está declinando em toda a Ásia, com exceção de Hong Kong. Em Osaka e Miyagi (Japão), o declínio é desprezível, tanto para os homens, quanto para as muIheres. Contudo, a mortalidade por câncer de estômago no Japão tem declinado com taxas de $20 \%$ a cada cinco anos. Os autores afirmam também que a disparidade entre as tendências da mortalidade e da incidência são conflitantes. Na Oceania, em todas a populações estudadas, há declínio tanto da incidência, quanto da mortalidade em ambos os sexos, embora haja diferenças significantes entre os valores dos coeficientes. Na Austrália, a mortalidade tem diminuído de $14 \%$ a $19 \%$ por período de cinco anos, taxas superiores às do Japão e da Nova Zelândia. Nas Américas, geralmente, há uma tendência a declínio ou estabilização tanto da incidência, quanto da mortalidade. A maioria das populações americanas analisadas mostram declínio da incidência. No Canadá, o decréscimo da mortalidade foi de $16 \%$ por período de cinco anos e, nos Estados Unidos, $11 \%$. Cuba, Porto Rico e Cali apresentam tendência de decréscimo na incidência. Para a mortalidade, os dados disponíveis do Chile, Costa Rica (20\% para cada cinco anos), Uruguai e Venezuela também mostram declínio das taxas.

As Tabelas 5 e 6 resumem os resultados de diversos trabalhos que analisam tendências de câncer de estômago no mundo, respectivamente para a incidência e para a mortalidade. Em todos eles há referência de declínio tanto da incidência, quanto da mortalidade por câncer de estômago.

Comparando-se com os resultados deste trabalho, verifica-se que, embora o comportamento da tendência de decréscimo da mortalidade por câncer de estômago no Brasil e nas capitais não seja uniforme, o que vem acontecendo aqui acompanha o que se observa em grande parte do mundo. Em grande parte das capitais analisadas, ainda se observa que a tendência de declínio provavelmente permanecerá por mais alguns anos (modelos linear e de segundo grau). Em outras, para as quais o modelo ajustado é o de terceiro grau, essa tendência já está mudando e, agora, aparentemente, os coeficientes se estabilizarão. Para a maioria, o decréscimo observado variou de $20 \%$ a $30 \%$ por período de cinco anos, padrão semelhante ao descrito acima por Coleman et al. (1993).

A análise da mortalidade por câncer de estômago para o Brasil e suas capitais, de 1977 a 1989, mostrou um declínio em praticamente todas essas localidades. O comportamento da mortalidade proporcional por esse tipo de câncer, dentre o total de neoplasias, mostrou que nas Regiões Norte, Nordeste e Centro-Oeste o estômago ainda é a localização de câncer mais importante na mortalidade por neoplasia no sexo masculino, ficando, geralmente, em terceiro lugar no sexo feminino, lembrando que se observou tendência de declínio nas capitais. Já no Sul e Sudeste, esse declínio foi mais acentuado e o câncer de estômago perdeu sua importância para o câncer de pulmão.

\section{Conclusões}

Em que pese a grande disparidade existente entre as capitais brasileiras, a tendência apresentada no período de 1977 a 1989 pela mortalidade por câncer do estômago é a de declínio, variando um pouco a forma com que se deu esse decréscimo nos coeficientes. A mortalidade por câncer de estômago ainda é importante como localização de câncer, principalmente nos 
Tendências da incidência de câncer de estômago em diversos países.

\begin{tabular}{|c|c|c|c|}
\hline local & autor(es) & período & tendência \\
\hline \multicolumn{4}{|l|}{ Europa } \\
\hline Oxford, Inglaterra & $\begin{array}{l}\text { Oxford Cancer Intel. } \\
\text { Unit, } 1994\end{array}$ & $\begin{array}{l}1979-1981 \\
\text { e } 1988-1990\end{array}$ & $\begin{array}{l}\text { Coeficiente masculino passoude } 18,5 \text { para } 15,8 \\
\text { e feminino de } 7,8 \text { para } 5,7 \text { (todos por cem mil } \\
\text { habitantes). }\end{array}$ \\
\hline Dinamarca & Storm et al., 1993 & $1945-1990$ & $\begin{array}{l}\text { Q ueda acentuada, estando em } 8 \text { o nos homens } \\
\text { e abaixo do } 10 \text { o nas mulheres. }\end{array}$ \\
\hline Suécia & $\begin{array}{l}\text { The Swedish Cancer } \\
\text { Registry, } 1993\end{array}$ & $1972-1991$ & $\begin{array}{l}\text { A taxa de decréscimo anual foi de } 3,2 \% \text { para os } \\
\text { homens e } 3,5 \% \text { para as mulheres. }\end{array}$ \\
\hline Zaragoza, Espanha & $\begin{array}{l}\text { Registro de Câncer } \\
\text { de Zaragoza, } 1986\end{array}$ & 1978-1982 & Declinando tanto nos homens quanto nas mulheres. \\
\hline noroeste da Hungria & J uhász, 1993 & $1962-1991$ & $\begin{array}{l}\text { Declínio para o sexo masculino, porém estacionário } \\
\text { no feminino. }\end{array}$ \\
\hline \multicolumn{4}{|l|}{ O ceania } \\
\hline O este da Austrália & Fitzgerald et al., 1994 & 1982-1991 & $\begin{array}{l}\text { Tendência de decréscimo de } 3,6 \% \text { ao ano para os } \\
\text { homens e não significante nas mulheres. }\end{array}$ \\
\hline Victoria (Austrália) & $\begin{array}{l}\text { Victorian Cancer } \\
\text { Registry, } 1991\end{array}$ & - & $\begin{array}{l}\text { Está declinando nos últimos anos e atualmente é o } \\
17 \text { o entre os cânceres em homens e } 10 \text { o nas mulheres. }\end{array}$ \\
\hline New South Wales (Austrália) & McCredie, 1992 & $1972-1989$ & $\begin{array}{l}\text { Decresceu } 24 \% \text { entre os homens e } 37 \% \text { entre } \\
\text { as mulheres. }\end{array}$ \\
\hline \multicolumn{4}{|l|}{ Américas } \\
\hline Porto Rico & $\begin{array}{l}\text { Registro Central de Cancer } \\
\text { de Porto Rico, } 1992\end{array}$ & $1955-1990$ & Tendência decrescente. \\
\hline $\begin{array}{l}\text { Diversas cidades } \\
\text { do Estado de Nova York }\end{array}$ & $\begin{array}{l}\text { New York S. C. } \\
\text { Register, } 1990\end{array}$ & Últimas décadas & $\begin{array}{l}\text { O declínio marcante observado nas últimas décadas. } \\
\text { já não existe. }\end{array}$ \\
\hline Canadá & $\begin{array}{l}\text { National Cancer Inst. } \\
\text { of Canada, } 1992\end{array}$ & $1980-1985$ & $\begin{array}{l}\text { Para os homens, o declínio é de } 1,8 \% \text { e para as } \\
\text { mulheres de } 2,7 \% \text { ao ano. }\end{array}$ \\
\hline
\end{tabular}

Tabela 6

Tendências da mortalidade por câncer de estômago em diversos países.

\begin{tabular}{|c|c|c|c|}
\hline local & autor(es) & período & tendência \\
\hline \multicolumn{4}{|l|}{ Europa } \\
\hline Oxford, Inglaterra & $\begin{array}{l}\text { Oxford Cancer Intel. } \\
\text { Unit, } 1994\end{array}$ & $\begin{array}{l}1979-1981 \\
\text { e } 1988-1990\end{array}$ & $\begin{array}{l}\text { Coeficiente masculino passou de } 12,8 \text { para } 9,6 \\
\text { e feminino de } 6,1 \text { para } 4,1 \text { (todos por cem mil } \\
\text { habitantes). }\end{array}$ \\
\hline \multicolumn{4}{|l|}{ Oceania } \\
\hline O este da Austrália & Fitzgerald et al., 1994 & 1982-1991 & $\begin{array}{l}\text { Tendência de decréscimo de } 2,1 \% \text { ao ano para os } \\
\text { homens e não significante nas mulheres. }\end{array}$ \\
\hline Victoria (Austrália) & $\begin{array}{l}\text { Victorian Cancer } \\
\text { Registry, } 1991\end{array}$ & - & $\begin{array}{l}\text { Está declinando nos últimos anos e atualmente é o } 4 \text { o } \\
\text { entre os cânceres em homens e } 6 \underline{0} \text { nas mulheres. }\end{array}$ \\
\hline New South Wales (Austrália) & McCredie, 1992 & 1972-1989 & Tendência de decréscimo, semelhante à incidência. \\
\hline \multicolumn{4}{|l|}{ Américas } \\
\hline Porto Rico & $\begin{array}{l}\text { Registro Central de Cancer } \\
\text { de Porto Rico, } 1992\end{array}$ & $1955-1990$ & Tendência decrescente. \\
\hline Uruguai & Vassalo, 1990 & 1963-1985 & $\begin{array}{l}\text { Única localização de câncer com tendência } \\
\text { decrescente (linear). }\end{array}$ \\
\hline Canadá & $\begin{array}{l}\text { National Cancer Inst. } \\
\text { of Canada, } 1992\end{array}$ & $1970-1988$ & $\begin{array}{l}\text { Para os homens, o declínio é de } 3,4 \% \text { e para } \\
\text { as mulheres de } 3,9 \% \text { ao ano. }\end{array}$ \\
\hline
\end{tabular}


homens, mas, talvez, nos próximos anos, isso venha a diminuir.

As tendências verificadas para esse período mostram-se semelhantes às observadas principalmente na Europa e lamenta-se não se possuirem séries históricas da incidência de câncer de estômago, para se avaliar se o decréscimo observado na mortalidade reflete a tendência da verdadeira incidência.

\section{Agradecimentos}

Agradeço a Profa Sabina L. D. Gotlieb por seus comentários e sugestões.

\section{Referências}

BOYLE, P. \& PARKIN, D. M., 1991. Statistical methods for registries. In: Cancer Registration - Principles and Methods (O. M. Jensen, D. M. Parkin, R. Maclennan, C. S. Muir \& R. G. Skeet, eds.), pp.126-158, Lyon: International Agency for Research on Cancer (IARC). IARC Scientific Publications, n. 95.

COLEMAN, M. P.; ESTEVE, J.; DAMIECKI, P.; ARSLAN, A. \& RENARD, H., 1993. Trends in Cancer Incidence and Mortality. Lyon: International Agency for Research on Cancer (IARC). IARC Scientific Publications, n. 121.

FITZGERALD, P.; THOMSON, N. \& THOMPSON, J., 1994. Cancer Incidence and Mortality in Western Australia, 1991. Statistical Series, n. 39, Health Department of Western Australia.

HAENSZEL, W. \& CORREA, P., 1975. Developments in the epidemiology of stomach cancer over the past decade. Cancer Research, 35:452-459.

HOWSON, C. P.; HIYAM A, T. \& WINDER, E. L., 1986. The decline in gastric cancer: epidemiology of an unplanned triumph. Epidemiologic Reviews, 8:127.

IBGE (FUNDAÇÃO INSTITUTO BRASI LEIRO DE GEOGRAFIA E ESTATÍSTICA), 1973. Censo Demográfico de 1970. Rio de Janeiro: IBGE.

IBGE (FUNDAÇÃO INSTITUTO BRASILEIRO DE GEOGRAFIA E ESTATÍSTICA), 1982. Censo Demográfico de 1980. Rio de Janeiro: IBGE.

JUHÁSZ, L., 1993. Cancer Incidence in the County Szabolcs - Szatmár-Bereg. Hungary 1990-91. Szatmár-Bereg: Cancer Series n. 14, Cancer Registry of the County Szabolcs.

KLEINBAUM, D. G.; KUPPER, L. L. \& MULLER, K. E., 1988. Applied Regression Analysis and other Multivariable Methods. Belmont, California: PWSKENT Publishing Company.
MCCREDIE, M.; HØYER, A.; COATES, M . \&TAYLOR, R., 1992. Trends in Cancer Incidence and Mortality in New South Wales: 1972-1989. New South Wales: Central Cancer Registry, NSW Cancer Council.

MELLO JORGE, M. H. P. \& LATORRE, M. R. D. O., 1994. Acidentes de trânsito no Brasil: dados e tendências. Cadernos de Saúde Pública, 10 (supl.1):1944.

MS (MINISTÉRIO DA SAÚDE), 1979/ 1993. Estatísticas de Mortalidade, Brasil, 1977/1989. Brasília: Divisão Nacional de Epidemiologia/MS.

MORETTIN, P. A. \& TOLOI, C. M., 1986. Séries Temporais. São Paulo: Atual Editora Ltda. Coleção Métodos Quantitativos.

NCIC (NATIONAL CANCER INSTITUTE OF CANADA), 1992. Canadian Cancer Statistics- 1990.

NEW YORK STATE CANCER REGISTRY, 1990. Time Trends in Cancer Incidence: 1977-1986. New York State Departament of Health.

OXFORD CANCER INTELLIGENCE UNIT, 1994. Cancer in the Oxford Region: 1979-1990.

REGISTRO DE CÂNCER DE ZARAGOZA, 1986. Incidencia del Cancer en Zaragoza, 1978-1982. Deputacion General de Aragon, Departamento de Sanidad, Bienestar Social y Trabajo.

REGISTRO CENTRAL DE CÂNCER DE PORTO RICO, 1992. Cancer en Puerto Rico, 1990.

SOUZA, E. R., 1994. Homicídios no Brasil: o grande vilão na década de 80. Cadernos de Saúde Pública, 10:45-60.

STORM, H. H.; MANDERS, T.; FRIIS, S. \& BRANDT, B., 1993. Cancer Incidence in Denmark - 1990. Danish Cancer Society.

THE SWEDISH CANCER REGISTRY, 1993. Cancer Incidence in Sweden-1991.

VASSALO, J. A., 1990. Cancer en el Uruguay, 1987. Registro Nacional de Cancer del Uruguay.

VICTORIAN CANCER REGISTRY, 1991. Statistical Re port- 1987. 